PROCEEDINGS OF THE

AMERICAN MATHEMATICAL SOCIETY

Volume 128, Number 5, Pages 1339-1346

S 0002-9939(00)05647-1

Article electronically published on February 7, 2000

\title{
REPRESENTATION OF FEEDBACK OPERATORS FOR PARABOLIC CONTROL PROBLEMS
}

\author{
BELINDA B. KING
}

(Communicated by John A. Burns)

\begin{abstract}
In this paper we present results on existence and regularity of integral representations of feedback operators arising from parabolic control problems. The existence of such representations is important for the design of low order compensators and in the placement of sensors. This paper extends earlier results of J. A. Burns and B. B. King to problems with $N$ spatial dimensions.
\end{abstract}

\section{INTRODUCTION AND MOTIVATION}

In this paper we consider the problems of existence and smoothness of functional gains for LQR feedback control systems governed by parabolic partial differential equations. It is well known that under suitable stabilizability conditions, a solution to this infinite dimensional LQR problem exists in the form of a bounded linear feedback operator $K$. The corresponding optimal control is given in feedback form

$$
u(t)=-K x(t) .
$$

Here, $K: X \rightarrow U$ maps the state space $X$ into the control space $U$ where $X$ is an infinite dimensional Hilbert space and, often, $U$ is the finite dimensional space $\mathbb{R}^{m}$ for some $m$. In this case the Riesz Representation Theorem provides an explicit representation of $K$ which is useful in the design and location of sensors (see [5]). If the control space $U$ is also an infinite dimensional Hilbert space, then the problem becomes much more difficult. However, provided that the algebraic Riccati equation has sufficiently smooth solutions, the corresponding feedback gain operator will have an explicit integral representation. The kernels of these integral operators are called functional gains and play a fundamental role in designing practical control laws (see [10, 14]).

This analysis is motivated by the need for specific representations for Riccati operators that can be used in designing sensors and actuators and in developing computational schemes for problems where the input and output operators are not Hilbert-Schmidt. This situation occurs in many boundary control problems and

Received by the editors August 17, 1995 and, in revised form, May 17, 1996.

1991 Mathematics Subject Classification. Primary 35K05, 47B99, 49J20.

Key words and phrases. Integral representation, Riccati operator, parabolic control problem.

This research was supported in part by the Air Force Office of Scientific Research under grant F49620-93-1-0280 while the author was a visiting scientist at the Air Force Center for Optimal Design and Control, Virginia Polytechnic Institute and State University, Blacksburg, Virginia 24061-0531, and by the National Science Foundation under grant DMS-9409506. 
in certain distributed control problems associated with optimal sensor/actuator placement.

In [12, Lupi, Chun, and Turner considered a distributed parameter LQR problem for an Euler-Bernoulli beam model. Their approach is interesting in that they make no prior assumptions regarding the form of the controls/actuators in an effort to make decisions about where actuators and sensors are best placed. In this vein, they assumed that the input operator was the identity. Miller and Van Schoor 14] considered the problem of constructing kernels for integral representations of feedback control laws obtained by solving LQR control problems for various beam models. They used these kernels to shape and design area averaging polyvinylidene fluoride sensors (a type of piezoelectric film). These sensors enable the real-time implementation of full state feedback for the infinite dimensional system governed by the Euler-Bernoulli beam equation (also, see 14]). However, as in 12, Miller and Van Schoor assumed the existence of an integral representation for the feedback control law and then proceeded to "approximate" these kernels by using finite element models. In both papers, fundamental mathematical questions concerning the existence of integral representations and the smoothness of the corresponding integral kernels are not considered. These issues are also important in the development and analysis of rigorous numerical approximations. Moreover, the ability to accurately compute these kernels is an essential component in the study of actuator/sensor placement.

In earlier papers by Rosen [15, 16] it was shown that, under suitable assumptions on the system input, output and weighting operators, the Riccati operator is Hilbert-Schmidt. This observation made it possible to develop an approximation theory in the space of Hilbert-Schmidt operators that can be used to analyze the convergence of numerical approximations. De Santis, Germani and Jetto in [7] consider general dynamical systems (not necessarily analytic semigroups), and then require that certain system operators (input, weighting, etc.) be nuclear. The problem we consider here lies between these two approaches, although it is more like that of Rosen [15. Our results herein apply to control problems with unbounded input operators.

Except for obvious cases with bounded input operators and a finite number of controllers, the problem of obtaining explicit representations of feedback laws is more complex than one might first imagine. In the most general case, this problem is equivalent to that which led Grothendieck to develop the theory of topological tensor products and nuclear spaces which led to the famous Schwartz Kernel Theorem. However, the fact that the operators of interest often arise as solutions to Riccati equations can be exploited to yield desired results. The representation problem for a one-dimensional parabolic problem with unbounded control operator was first considered in [4] and [9]. Therein, it was shown that as long as the input operator is bounded relative to the open loop dynamic operator, the solution to the algebraic Riccati equation is Hilbert-Schmidt. This fact was then exploited to show that the resulting feedback operator had an integral representation.

Here we consider a specific problem governed by the heat equation in $\mathbb{R}^{N}$ and allow rather general input operators. In particular, we do not require that the input operator be Hilbert-Schmidt (or even bounded) and we allow for non-compact weighting operators. We show that it is the specific structure of the parabolic problem that provides the smoothness needed to prove the existence of functional gains. Although the one-dimensional results are established in [4, 9], the extension 
of those results to more than one space dimension is not trivial and is the goal of this note.

To provide a framework for analysis, the general control problem for a parabolic partial differential equation can be written in abstract form as

$$
\dot{x}(t)=A x(t)+B u(t), \quad x(0)=x_{0}
$$

where at time $t$, the state $x(t)$ lies in a Hilbert space $X$ (i.e., the state space). The control input, $u(t)$, lies in the input space $U$. A standard approach to computing a control is the linear quadratic regulator (LQR) problem; the LQR problem consists of finding a $u_{\text {opt }}$ which minimizes the cost functional

$$
J(x, u)=\int_{0}^{\infty}\left[\langle Q x(t), x(t)\rangle_{X}+\langle R u(t), u(t)\rangle_{U}\right] d t
$$

subject to satisfying the dynamics of (1.1). Under certain conditions on the operators $A, B, Q, R$ and initial condition, $x_{0}$ (see [3]), it follows that $u_{o p t}$ exists and has the form

$$
u_{\text {opt }}(t)=-K x(t)
$$

where the feedback gain operator $K: X \rightarrow U$ is given by

$$
K=R^{-1} B^{*} \Pi
$$

and $\Pi$ satisfies the algebraic Riccati equation (ARE) in $X$

$$
\langle\Pi x, A y\rangle_{X}+\langle A x, \Pi y\rangle_{X}-\left\langle R^{-1} B^{*} \Pi x, B^{*} \Pi y\right\rangle_{U}+\langle Q x, y\rangle_{X}=0,
$$

for all $x, y$ in $\mathcal{D}(A)$. Here $Q=Q^{*} \geq 0, R=R^{*}>0$ and equation (1.5) holds in the weak sense (see [1]).

We limit our discussion to control systems satisfying the following hypothesis:

(H) The spaces $X$ and $U$ are separable Hilbert spaces and

(i) the linear operator $A$ is the generator of a $C_{0}$-semigroup $S(t)$ on $X$ and there exist $M>0, \omega>0$ such that $\|S(t)\| \leq M e^{-\omega t}$,

(ii) the operator $Q: X \rightarrow X$ is bounded, $R=I_{U}$,

(iii) the (possibly unbounded) linear operator $B$ maps $U$ into $\left[\mathcal{D}\left(A^{*}\right)\right]^{\prime}$. Moreover, there exists $\gamma$ with $0 \leq \gamma<1$, such that $A^{-\gamma} B \in \mathcal{L}(U, X)$.

In [7, 15, 16] it is assumed that $Q$ and $B$ are bounded linear operators. Rosen (in [15]) assumes that $A$ is strongly coercive, $\Pi B R^{-1} B^{*} \Pi$ is Hilbert-Schmidt whenever $\Pi$ is Hilbert-Schmidt and that $Q$ is Hilbert-Schmidt. On the other hand, De Santis, Germani and Jetto (in [7]) make no additional assumptions on $A$, but require that $Q$ be nuclear and $B$ be bounded from $U$ to $X$. Hence, the assumption on $Q$ in [7] is stronger than the corresponding condition in [15]. The two problems are not mutually exclusive and, as one might expect, there is no unified theory.

In this paper we consider problems with $B$ unbounded and $Q$ not HilbertSchmidt. Unbounded $B$ operators allow us to treat certain boundary control problems and problems involving piezoelectric actuators/sensors. Also, the case where $Q=B=I_{X}$ (the identity on $X$ ) arises naturally in the solution of optimal sensor/actuator location problems (see [4, 6, 10, 12]). In addition, note that for the parabolic control problem above, $A$ satisfies $(\mathbf{H})$ and generates an analytic semigroup, a fact which is important in the proof of our main result. This property and the following theorem may be found in [11]. 
Theorem 1. Assume that hypothesis $(\mathbf{H})$ holds. If A generates an analytic semigroup, then there exists a self-adjoint, bounded, non-negative definite linear operator $\Pi=\Pi^{*}$ that solves the ARE (1.5). Moreover,

(a) For each $\epsilon>0$, the operator $\left[A^{*}\right]^{1-\epsilon} \Pi$ belongs to $\mathcal{L}(X, X)$.

(b) If $A$ is self-adjoint, normal or has a Riesz basis of eigenvectors, then $\epsilon$ can be taken to be 0 .

(c) The operator $B^{*} \Pi$ belongs to $\mathcal{L}(X, U)$.

Observe that if $A$ has a compact resolvent, then $\Pi$ is compact. In general it is not possible to conclude that $\Pi$ has additional smoothing properties unless more is known about the operators $A, B, Q$ and $R$. Consider the following example:

Example 1. Let $X=U=L_{2}(0,1)$ and set $A=Q=R=I_{L_{2}}$. If $B=\sqrt{3} I$, then $\Pi=I$ is the unique positive-definite solution to the ARE (1.5). Hypothesis $(\mathbf{H})$ holds and $A$ generates an analytic semigroup. However, $\Pi$ is not compact.

\section{Representation of LineAR OpERATORS With RANGE IN $H^{\alpha}(\Omega)$}

In our treatment of the one-dimensional parabolic problem in [9], we made use of a classical theorem due to Fullerton (see [8], Theorem 6). This theorem gives representations of operators on $L_{2}[0,1]$ with range in $C^{m}$. We now extend this theorem to hold for operators on $L_{2}(\Omega), \Omega \in \mathbb{R}^{N}$, with range in the Sobolev space $H^{\alpha}(\Omega)$, which, using the Sobolev embedding theorems (see [1]), are embedded in $C$ for $\alpha>\frac{N}{2}$.

We assume that $\Omega \subset \mathbb{R}^{N}$ is a bounded set having the strong local Lipschitz property (see [1]). Maurin in [13] proved that if $m$ and $k$ are non-negative integers with $k>\frac{N}{2}$, then the Sobolev imbedding $H^{m+k}(\Omega) \rightarrow H^{m}(\Omega)$ is Hilbert-Schmidt. The extension to fractional order Sobolev spaces was given by Wloka in [19] as the following result.

Proposition 1. If $0 \leq \alpha<\beta$ are real numbers, then the Sobolev imbeddings

$$
H^{\beta+\frac{N}{2}}(\Omega) \rightarrow H^{\alpha}(\Omega)
$$

are Hilbert-Schmidt. In particular, the imbedding

$$
\Lambda_{\beta}: H^{\beta+\frac{N}{2}}(\Omega) \rightarrow H^{0}(\Omega)=L_{2}(\Omega)
$$

is Hilbert-Schmidt for all $\beta>0$.

We apply Proposition 1 to obtain the representation theorem.

Theorem 2. If $\beta>0$ and $T: L_{2}(\Omega) \rightarrow H^{\beta+\frac{N}{2}}(\Omega)$ is a bounded linear operator, then there exists a kernel $k(\cdot, \cdot)$ defined on $\Omega \times \Omega$ such that

(i) $k(\cdot, \cdot) \in L_{2}(\Omega \times \Omega)$,

(ii) $[T \phi](\xi)=\int_{\Omega} k(\xi, y) \phi(y) d y$,

(iii) $\|T\|_{2}^{2}=\int_{\Omega \times \Omega}|k(\xi, y)|^{2} d \xi d y$.

Proof. It follows from Proposition 1 that the Sobolev imbedding $\Lambda_{\beta}: H^{\beta+\frac{N}{2}}(\Omega) \rightarrow$ $L_{2}(\Omega)$ is Hilbert-Schmidt. Let $\hat{T}=\Lambda_{\beta} \circ T$ be the operator $T$ as an operator from $L_{2}(\Omega)$ to $L_{2}(\Omega)$. Since $\hat{T}$ is the composition of a bounded operator with a HilbertSchmidt operator, $\hat{T}$ is also Hilbert-Schmidt. 
It now follows from Theorem VI.23 in 17] that there is a $k(\cdot, \cdot) \in L_{2}(\Omega \times \Omega)$ such that for all $\phi \in L_{2}(\Omega)$

$$
[T \phi](\xi)=[\hat{T} \phi](\xi)=\int_{\Omega} k(\xi, y) \phi(y) d y .
$$

Moreover, it is clear that

$$
\|T\|_{2}^{2}=\|\hat{T}\|_{2}^{2}=\int_{\Omega \times \Omega}|k(\xi, y)|^{2} d \xi d y
$$

and this completes the proof.

\section{Control of Parabolic systems}

Here we assume that $N=1,2$ or 3 and that $\Omega \subseteq \mathbb{R}^{N}$ is a smooth bounded set with boundary $\Gamma$. Let $X=L_{2}(\Omega)$ and define the operator $A$ on the domain

$$
\mathcal{D}(A)=H^{2}(\Omega) \cap H_{0}^{1}(\Omega),
$$

by

$$
A x=\Delta_{\xi} x+c^{2} x=\sum_{i=1}^{N} \frac{\partial^{2} x}{\partial \xi_{i}^{2}}+c^{2} x .
$$

The controlled heat equation can be written as

$$
\dot{x}(t)=A x(t)+B u(t)
$$

where $B$ is assumed to satisfy condition $(\mathbf{H})$-(iii). The constant $c^{2}$ is selected such that $0 \in \rho(A)$.

We note that this framework includes certain boundary and point control problems of Dirichlet type (see [3, 11]). It is shown in [1] that $A$ is self-adjoint and generates an analytic semigroup on $L_{2}(\Omega)$.

Let $Q=I_{L_{2}(\Omega)}$ and $R=I_{U}$ be the identity operators on $X$ and $U$ respectively. The extension to more general operators is straightforward. We observe that $Q$ is not compact and hence the results in [7, 15] do not apply. The LQR problem is to minimize

$$
J(u)=\int_{0}^{\infty}\left[\int_{\Omega}|x(t, \xi)|^{2} d \xi+|u(t)|_{U}^{2}\right] d t
$$

over all controls $u \in L_{2}([0, \infty] ; U)$.

Since hypothesis $(\mathbf{H})$ holds and $A$ is a self-adjoint generator of an analytic semigroup on $L_{2}(\Omega)$, Theorem 1 applies with $\epsilon=0$. Moreover, one has the following result.

Theorem 3. If $\Pi=\Pi^{*}$ is the unique non-negative solution of the (ARE), (1.5), for the parabolic control problem (3.1)-(3.4), then $\Pi$ is Hilbert-Schmidt. In particular, there exists a kernel function $k(\cdot, \cdot)$ such that

(a) $k(\cdot, \cdot) \in L_{2}(\Omega \times \Omega)$,

(b) $k(\xi, y)=k(y, \xi)$ for $\xi, y \in \Omega$,

(c) $\int_{\Omega \times \Omega} \phi(\xi) k(\xi, y) \phi(y) d \xi d y$ for all $\phi \in L(\Omega)$,

(d) $[\Pi \phi](\xi)=\int_{\Omega} k(\xi, y) \phi(y) d y$ for all $\phi \in L_{2}(\Omega)$. 
Proof. By Theorem 1, it follows that $A \Pi \in \mathcal{L}\left(L_{2}(\Omega), L_{2}(\Omega)\right)$. Let $W=\mathcal{D}(A)$ with norm $\|\phi\|_{W}=\|A \phi\|_{L_{2}(\Omega)}$. Since $A$ is positive, $W$ is norm equivalent to $H_{2}(\Omega)$ so there exists a constant $c_{1}$ such that for each $\phi \in \mathcal{D}(A)=W$

$$
\|\phi\|_{H_{2}(\Omega)} \leq c_{1}\|\phi\|_{W}=c_{1}\|A \phi\|_{L_{2}(\Omega)}
$$

(see [18], p. 53). Since $A \Pi$ is bounded on $L_{2}(\Omega)$, there is a constant $c_{2}$ such that if $\phi \in L_{2}(\Omega)$, then $\Pi \phi \in \mathcal{D}(A) \subseteq H^{2}(\Omega)$ and

$$
\|A(\Pi \phi)\|_{L_{2}(\Omega)} \leq c_{2}\|\phi\|_{L_{2}(\Omega)} .
$$

It follows that if $\phi \in L_{2}(\Omega)$, then $\Pi \phi \in H^{2}(\Omega)$ and

$$
\|\Pi \phi\|_{H^{2}(\Omega)} \leq c_{1}\|A(\Pi \phi)\|_{L_{2}(\Omega)} \leq c_{1} c_{2}\|\phi\|_{L_{2}(\Omega)} .
$$

Therefore, the operator $\Pi: L_{2}(\Omega) \rightarrow H^{2}(\Omega)$ is bounded.

Let $\beta=\left(2-\frac{N}{2}\right)>0$. Theorem 2 yields the existence of a kernel $k(\cdot, \cdot)$ satisfying (a) and (d). Moreover, since $\Pi=\Pi^{*}$ it follows that $k(\xi, y)=k(y, \xi)$ and (b) holds. Condition (c) is a direct consequence of the inequality

$$
\langle\phi, \Pi \phi\rangle \geq 0,
$$

which holds because $\Pi$ is non-negative.

In order to obtain an integral representation of the feedback operator $k=B^{*} \Pi$, additional assumptions are needed on $B$.

Corollary 1. Assume that $A$ is defined by (3.1)-(3.2), and $Q$ and $R$ are the identity operators on $L_{2}(\Omega)$ and $U$, respectively. If $B: U \rightarrow L_{2}(\Omega)$ is bounded, then $k=$ $-B^{*} \Pi$ is Hilbert-Schmidt. In particular, if $U=L_{2}\left(\Omega^{\prime}\right)$, then there exists a kernel $h(\cdot, \cdot) \in L_{2}\left(\Omega^{\prime} \times \Omega\right)$ such that for $\phi \in L_{2}(\Omega)$

$$
[k \phi](\xi)=\int_{\Omega} h(\xi, y) \phi(y) d y .
$$

Proof. This result follows from the observation that $B^{*} \circ \Pi$ is Hilbert-Schmidt and results regarding representation of Hilbert-Schmidt operators from $L_{2}(\Omega)$ to $L_{2}\left(\Omega^{\prime}\right)$ (see [2], Theorem 2, p. 277).

Observe that this is similar to the results in [15]; however, it is not required that we assume anything about the operator $Q$. In certain cases one can obtain explicit representations for unbounded $B$ operators. In the one-dimensional case, the kernel $k(\cdot, \cdot)$ in the representation for $\Pi$ for the heat equation belongs to $C^{1}([a, b] \times[a, b])$. Thus, the kernel is smooth and symmetric (see [4, 9]).

Example 2. Let $\Omega=[a, b]$ and let $\partial_{m}$ be the maximal differential operator defined by $\mathcal{D}\left(\partial_{m}\right)=H^{1}(a, b)$ and $\left[\partial_{m} \phi\right](\xi)=-\frac{d}{d \xi} \phi(\xi)$. The adjoint operator, $\partial_{m}^{*}$, is the minimal operator $\partial_{m}^{*}=\partial_{0}$ defined on the domain $H_{0}^{1}(a, b)$ by $\left[\partial_{0} \phi\right](\xi)=\frac{d}{d \xi} \phi(\xi)$. The extension of $\partial_{m}$ from $L_{2}(a, b)$ to $H^{-1}(a, b) \subseteq\left(\mathcal{D}\left(\partial_{m}\right)\right)^{\prime}$ is the input operator $B$ and hypothesis $(\mathbf{H})$ holds. Moreover, since

$$
[\Pi \phi](\xi)=\int_{a}^{b} k(\xi, y) \phi(y) d y
$$

where $k(\cdot, \cdot) \in C^{1}([a, b] \times[a, b])$, it follows that the feedback operator $K=B^{*} \Pi$ has the representation

$$
\left[B^{*} \Pi \phi\right](\xi)=\int_{a}^{b} \frac{\partial}{\partial \xi} k(\xi, y) \phi(y) d y
$$


Clearly, if $B=[-A]^{\gamma}$ with $\gamma \leq \frac{1}{2}$, then one has a similar representation

$$
\left[B^{*} \Pi \phi\right](\xi)=\int_{a}^{b} h(\xi, y) \phi(y) d y
$$

with $h(\xi, y) \in L_{2}([a, b] \times[a, b])$. The numerical results in [4, 9] suggest a much stronger result. Namely, that (3.6) holds for $B=[-A]^{\gamma}$ with $\gamma<1$. We leave this as a conjecture.

\section{Conclusions}

In this paper, we have extended results for existence and regularity of integral representations of Ricatti operators to $N$-dimensional parabolic problems. We obtained the desired results by making use of the analytic semigroup arising from the openloop dynamics of the problem, rather than restricting allowable types of input operators as in previous work [15, 16]. Results of this type for one-dimensional hyperbolic control problems were given in $[5$. For that problem, these types of results are more intricate, highly dependent on the type of damping, and are not yet complete. Moreover, they do not easily extend to results for problems in higher spatial dimensions as do the results presented here. This problem will be addressed in a future paper.

\section{REFERENCES}

[1] R. A. Adams, Sobolev Spaces, Academic Press, New York, 1975. MR 56:9247]

[2] J. P. Aubin, Applied Functional Analysis, John Wiley \& Sons, New York, 1979. MR 81a:46083

[3] A. Bensoussan, G. DaPrato, M.C. Delfour, and S. Mitter Representation and Control of Infinite Dimensional Systems, Vol I. \& II, Birkhäuser, Boston, 1993. MR 94b:49003. MR 94m:49001

[4] J. A. Burns and B. B. King, "A Note on the Regularity of Solutions of Infinite Dimensional Riccati Equations," Appl. Math. Lett., v. 7, 1994, pp. 13-17. MR 96b:93057

[5] J. A. Burns and B. B. King, "Optimal Sensor Location for Robust Control of Distributed Parameter Systems," Proc. of the 33rd IEEE Control and Decision Conference, Dec. 1994, pp. 3967-3972.

[6] J.A. Burns and B.B. King, "Representation of Feedback Operators for Hyperbolic Systems", Proc. Computation and Control IV, Birkhäuser, 1995. MR 96c:93006

[7] A. De Santis, A. Germani and L. Jetto, "Approximation of the Algebraic Riccati Equation in the Hilbert Space of Hilbert-Schmidt Operators," SIAM J. Control Optim., v. 31, 1993, pp. 847-874. MR 94m:47128

[8] R. E. Fullerton, "Linear Operators with Range in a Space of Differentiable Functions," Duke Math. Journal, v. 13, 1946, pp. 269-280. MR 8:32e

[9] B. B. King, "Existence of Functional Gains for Parabolic Control Systems" Proc. Computation and Control IV, Birkhäuser, 1995. MR 96f:93044

[10] C. S. Kubrusly and H. Malebranche, "Sensors and Controllers Location in Distributed Systems: A Survey," Automatica, v. 21, 1985, p. 117-128. [MR 86e:93059

[11] I. Lasiecka and R. Triggiani, Differential and Algebraic Riccati Equations with Application to Boundary/Point Control Problems: Continuous Theory and Approximation Theory, Lecture Notes in Control and Information Sciences, v. 164, Springer-Verlag, Berlin, Heidelberg, 1991. MR 92k:93009

[12] V. D. Lupi, H. M. Chun, J. D. Turner, "Distributed Control without Mode Shapes or Frequencies," Adv. in the Astro. Sci., v. 76, 1991, pp. 447-470.

[13] K. Maurin, "Abbildungen vom Hilbert-Schmidtschen Typus und ihre Anwendungen", Math. Scand., v. 9, 1961, p. 359-371. MR 25:4364

[14] D. W. Miller and M. C. Van Schoor, "Formulation of Full State Feedback for Infinite Order Structural Systems," Proc. 1st US/Japan Conf. on Adapt. Struct., Maui, Hawaii, 1990, pp. 304-331. 
[15] I. G. Rosen, "On Hilbert-Schmidt Norm Convergence of Galerkin Approximation for Operator Riccati Equations," Int. Series of Numer. Math. 91, Birkhäuser, Basel, 1989, pp. 335-349. MR 91e:65085

[16] I. G. Rosen, "Convergence of Galerkin Approximations for Operator Riccati Equations - A Nonlinear Evolution Equation Approach," J. Math. Anal. Appl., v. 155, 1991, pp. 226-248. MR 91m:65169

[17] M. Reed and B. Simon, Functional Analysis I, Academic Press, New York, 1980. MR 58:12429a (1972 edition)

[18] R. Temam, Infinite-Dimensional Dynamical Systems in Mechanics and Physics, SpringerVerlag, New York, 1988. MR 89m:58056

[19] J. Wloka, "Vektorvertige Sobolev-Slobodeckijsche Distributionen," Math. Zeitschr., v. 98, 1967, pp. 303-318. MR 35:7122

Department of Mathematics, Oregon State University, Corvallis, Oregon 97331-4605 Current address: Interdisciplinary Center for Applied Mathematics, Virginia Tech, Blacksburg, Virginia 24061-0531

E-mail address: bbking@icam.vt.edu 